\title{
IRAS 18576+0341: A new addition to the class of Galactic Luminous Blue Variables
}

\author{
A. Pasquali ${ }^{1}$ and F. Comerón ${ }^{2}$ \\ 1 ESO/ST-ECF, Karl-Schwarzschild-Strasse 2, 85748 Garching bei Muenchen, Germany \\ e-mail: apasqual@eso.org \\ 2 ESO, Karl-Schwarzschild-Strasse 2, 85748 Garching bei Muenchen, Germany \\ e-mail: fcomeron@eso.org
}

Received 11 October 2001 / Accepted 16 November 2001

\begin{abstract}
We present $J H K$ photometry and low-resolution $H K$ spectroscopy of IRAS $18576+0341$, a galactic Luminous Blue Variable (LBV) candidate. The star is heavily reddened with observed magnitudes $J=13.21, H=$ 9.88 and $K=8.06$. These values and previously published photometry define a lightcurve with an amplitude of $\simeq 1.4$ mag between 1999 June and 2001 August, quite compatible with what is observed for well-studied LBVs, i.e. AG Carinae and HR Carinae. The spectrum of IRAS $18576+0341$ is characterised by prominent H, He and Fe emissions, which are recurrent features in the spectra of well known LBVs such as He 3-519. These findings together with the recent detection of an associated circumstellar nebula (Ueta et al. 2001) confirm the LBV nature of IRAS $18576+0341$.
\end{abstract}

Key words. stars: emission line, circumstellar matter, outflows

\section{Introduction}

The upper part of the Hertzprung-Russell diagramme is populated by a variety of massive stars at different evolutionary stages. Our current knowledge of stellar evolution sorts them into few evolutionary phases where the evolution is mainly driven by stellar winds and mass loss. Langer et al. (1994) showed that for stellar initial masses larger than 30-40 $M_{\odot}$ O stars evolve off the main sequence and end up as Wolf-Rayets by losing $\geq 50 \%$ of their mass. It is widely recognized that the highest mass-loss rate (up to $10^{-3} M_{\odot} / \mathrm{yr}$ ) occurs in a transition phase called Luminous Blue Variable.

At this point of their evolutionary path, massive stars are at the end of the $\mathrm{H}$ core burning (Pasquali et al. 1997) and close to the $\Omega$ limit (Langer \& Heger 1998) when the combination of the Eddington luminosity with the surface rotation rate makes the stellar envelope unstable. As a result, LBVs undergo giant eruptions and minor bursts, producing N-enriched circumstellar nebulae (Smith et al. 1998). They are also characterised by spectrophotometric variability, with periods of a few years and amplitudes of 1-2 mag in $V$. At minimum LBVs spectroscopically resemble Ofpe/WN9 stars while at maximum they mimic A supergiants (Humphreys \& Davidson 1994). $\eta$ Carinae and P Cygni are the best known examples of LBVs.

Send offprint requests to: A. Pasquali, e-mail: apasqual@eso.org
So far, LBV nebulae have been extensively studied in the optical regime which has allowed a detailed analysis of their chemistry, morphology and kinematics. Expansion velocities indicate a dynamical age of a few $10^{4}$ years which points to a very short-lived phase. Indeed, LBVs are rare objects: only 40 confirmed LBVs are known in the Local Group so far.

On top of this, the LBV demography is constrained by selection biases. Indeed, optical detection has so far been the main "discovery channel". Therefore, it is likely that LBVs have been missed in heavily reddened regions of the Galaxy. This is the case for a handful of LBVs which were first detected by Figer et al. (1999) and Geballe et al. (2000) in the Quintuplet cluster through near-infrared imaging and spectroscopy.

Another case is offered by G079.29+0.46, which was observed with optical and infrared spectroscopy by Voors et al. (2000). The nature of the detected emission lines has confirmed the LBV classification. The spectra have also showed the presence of a circumstellar nebula.

Very recently, an unidentified IR source, IRAS $18576+0341$, has been observed by Ueta et al. (2001) at mid-infrared wavelengths. The authors have resolved a circumstellar nebula with a diameter $\geq 7^{\prime \prime}$. The images taken at $10.3 \mu \mathrm{m}$ and $18.0 \mu \mathrm{m}$ show an elongated shell with two bright lobes at the opposite ends of its major axis. The dust temperature is between 115 and $135 \mathrm{~K}$ and the dust appears to be dominated by silicates 
with a minor contribution from PAHs. Radiative transfer models have been used to derive the central star temperature and luminosity: $15000 \pm 9000 \mathrm{~K}$ and $10^{6.4} L_{\odot}$. The stellar parameters together with the detection of a circumstellar, elliptical shell make IRAS $18576+0341$ a suspected LBV. We have performed IR photometry and low-resolution spectroscopy of the star in order to clarify the nature of IRAS $18576+0341$.

\section{Observations}

We observed IRAS $18576+0341$ on 2001 August 28 and 29, with the near-infrared imager and spectrograph MAGIC mounted on the $1.23 \mathrm{~m}$ telescope of the German-Spanish Astronomical Center in Calar Alto (Spain). This instrument uses a NICMOS3 $256 \times 256$ pixel $^{2}$ detector yielding a scale of $11^{\prime \prime} 15$ per pixel in imaging mode.

Imaging of the field centered at the position of IRAS $18576+0341$ was performed on 2001 August 29 in the $J, H$, and $K M$ filters by using a dither pattern of 9 points on a $3 \times 3$ grid with $40^{\prime \prime} \times 40^{\prime \prime}$ point spacing, adding up 60 integrations of $1 \mathrm{~s}$ each at every grid position. Specifically, the $K M$ filter covers the spectral range between 1.9 and $2.3 \mu \mathrm{m}$, while the classical $K$ band spans the 2.0-2.4 $\mu \mathrm{m}$ interval. A sky frame was constructed for each field and filter by median-filtering its component frames uncorrected for the motions of the telescope between consecutive grid points, so as to remove stellar images. The sky frame was subtracted from each image in the dither, and the resulting sky-subtracted and flat-fielded images were then aligned and coadded using the stellar images as reference. This procedure was repeated four times per filter with the center of the dither in slightly different positions. The four reduced frames were then combined into a single, final image in each filter. All the data reduction was performed using standard IRAF tasks and dedicated scripts. Photometric zeropoints in each filter were obtained by observing the infrared standard star P530-D (Persson et al. 1998) on the same night. Both IRAS $18576+0341$ and P530-D were observed when they were crossing the meridian near the zenith.

Aperture photometry on the images was performed using IRAF scripts that made use of the DAOFIND and PHOT tasks (Stetson 1987). Due to the crowded field of IRAS $18576+0341$, photometry was carried out using undersized apertures as compared to the size of the pointspread function (PSF). A correction factor for the loss of flux outside the aperture was computed from the brightest unsaturated and relatively isolated stars in the field by using larger apertures, and then applied to each detected star.

Spectroscopy of IRAS $18576+0341$ was performed on 2001 August 28 using a grism covering both the $H$ and $K$ bands in a single setup, in combination with a slit yielding a width of 2 pixels on the detector. This setup allowed us to obtain spectra covering the $1.45-2.40 \mu \mathrm{m}$ range at a resolution of $R \simeq 240$. We obtained six spectra at positions evenly spaced along the slit, each one being the sum

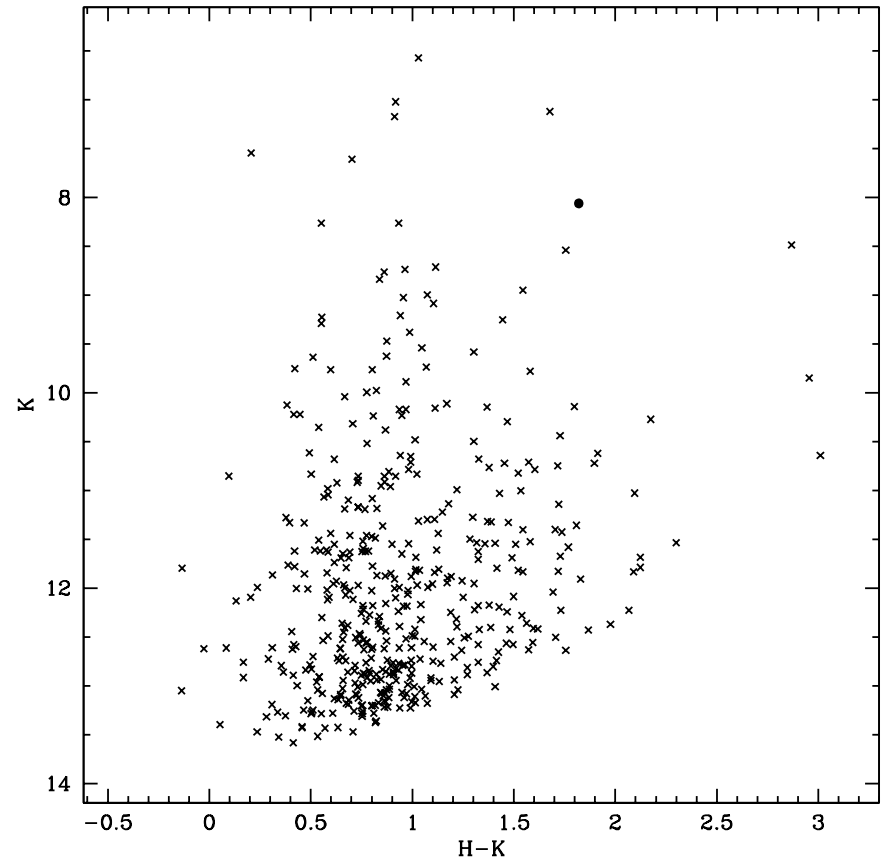

Fig. 1. The observed $(H-K)$ vs. $K$ diagramme of the field centered on IRAS $18576+0341$, here represented with a filled circle.

of 10 exposures of $6 \mathrm{~s}$ each. We also obtained spectra of the G5IV star HD 109711 (assumed to be nearly featureless at that resolution) in the same conditions just before observing IRAS $18576+0341$, in order to enable the removal of telluric features. For spectrum extraction, each pair of consecutive frames were differenced in order to cancel both the sky emission and the background level, and then the differenced frames were flat-fielded and spectra extracted using the APALL task under IRAF. Individual spectra obtained at each position along the slit were wavelengthcalibrated using OH airglow lines (Oliva \& Origlia 1992). The individual, wavelength-calibrated spectra were then combined separately for IRAS $18576+0341$ and for the telluric reference star and ratioed. The result was normalized to the fitted continuum.

\section{The results}

\subsection{IR photometry}

The observed colour-magnitude and colour-colour diagrammes of the $5^{\prime} \times 5^{\prime}$ field centered on IRAS $18576+0341$ are shown in Figs. 1 and 2. Here, IRAS $18576+0341$ is represented with a filled circle at $J=13.21 \pm 0.1, H=$ $9.88 \pm 0.05$ and $K=8.06 \pm 0.05$. The appearance of the diagrammes is typical of a randomly selected field in the direction of the Milky Way. The loci of unreddened giants and dwarfs (Bessell \& Brett 1988) are plotted in the colour-colour diagramme, as well as a reddening vector following the extinction law of Rieke \& Lebofsky (1985). The reddening vector plotted here has its origin at the position of a B0 star and its length corresponds to the shift in the diagramme produced by a visual extinction 


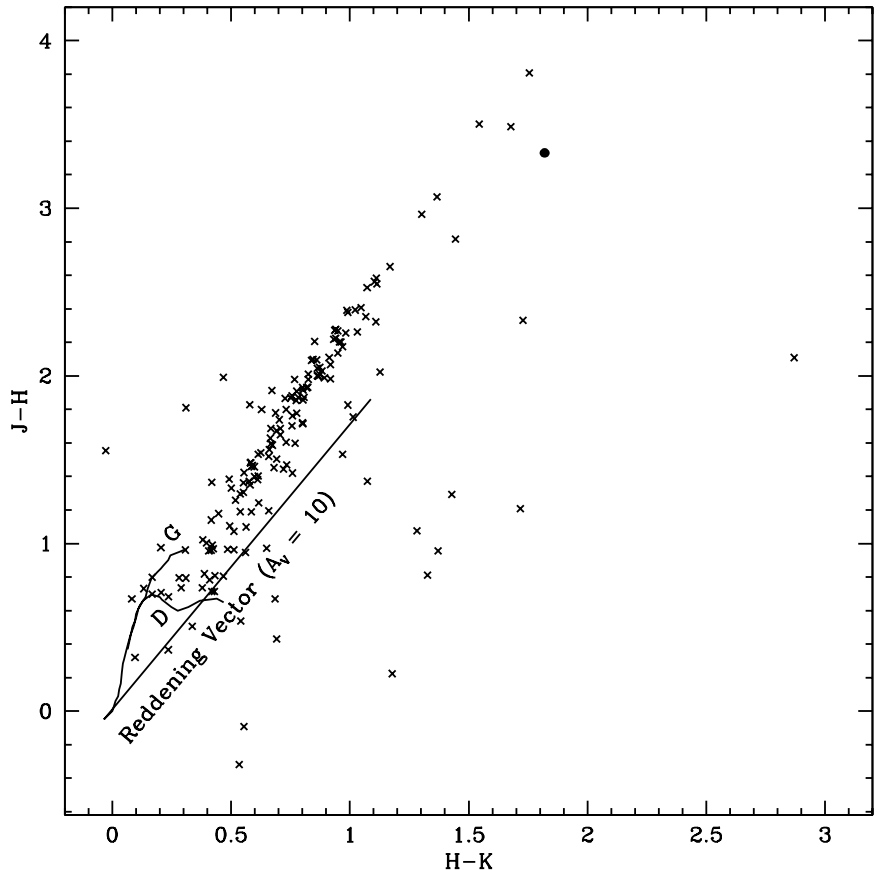

Fig. 2. The observed $(H-K)$ vs. $(J-H)$ diagramme of the field centered on IRAS $18576+0341$, here represented with a filled circle. The letters D and G indicate the loci of unreddened dwarfs and giants, respectively. The reddening vector, corresponding to $A_{V}=10 \mathrm{mag}$, is also plotted.

of $A_{V}=10$ mag. Assuming typical early-type colours for IRAS $18576+0341$ we derive an extinction of $A_{V}=17 \mathrm{mag}$ in its direction. Ueta et al. (2001) have estimated the interstellar $A_{V}$ to be between 24 and 32 mag.

We examined the spatial distribution of sources having $H-K$ colours similar (to within $0.2 \mathrm{mag}$ ) to those of IRAS 18576+0341, without finding any obvious evidence for clustering that may indicate the existence of other bright stars physically related to the LBV candidate. The majority of sources deviating from the band defined by reddened stars with normal colours were found to have close companions along the line of sight contaminating the photometry. The few ones for which contamination was not the cause for such colours are scattered uniformly in the field of view, again without signs of clustering towards IRAS $18576+0341$. The same can be said about the reddest and brightest sources appearing in the colourmagnitude diagramme.

Garcia-Lario et al. (1997) and Ueta et al. (2001) monitored IRAS $18576+0341$ in the JHK bands. We have included their photometries in our dataset and plotted the infrared lightcurves of the star in Fig. 3. Given the gap in the photometric monitoring of the star between 1989 May and 1999 June, only the points later than 1999 June have been connected with solid lines. If we restrict our analysis to the years between 1999 and 2001, we can see that the star was near minimum in $H$ and $K$ in 2000 August; it faded by $\simeq 1.4$ mag in 1.17 years from 1999 June to 2000 August. In 2001 August IRAS 18576+0341 has

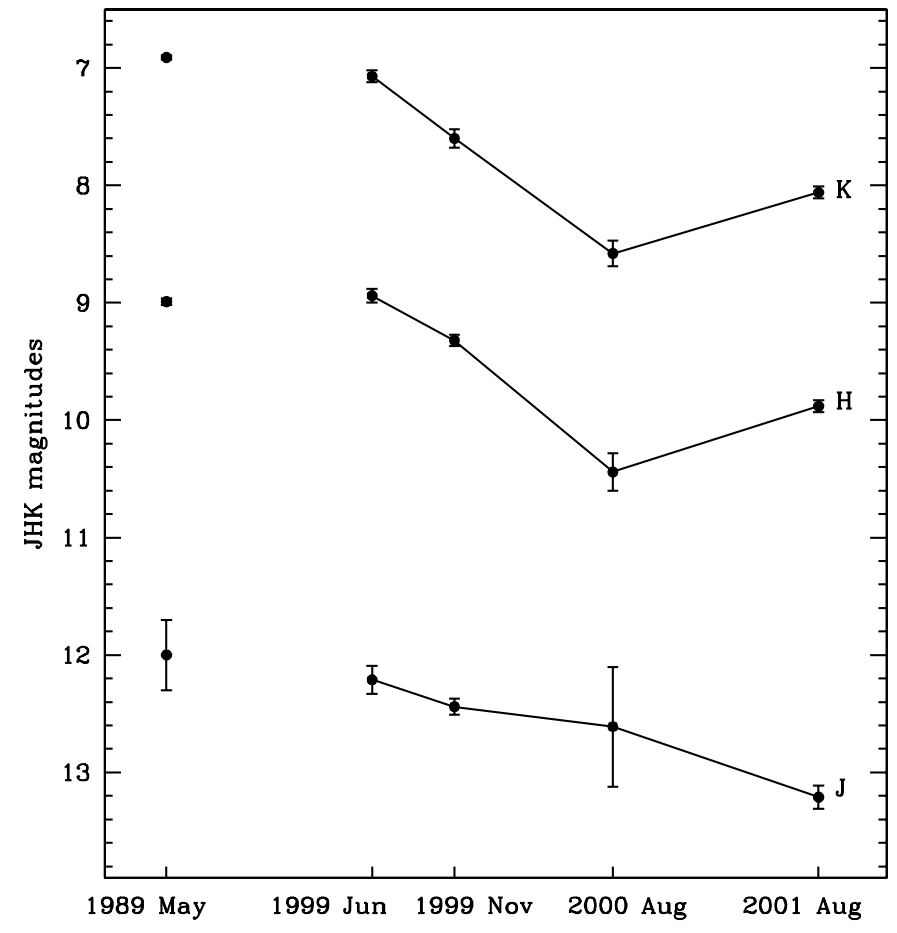

Fig. 3. The reddened JHK lightcurves of IRAS $18576+0341$ observed from 1989 May to 2001 August. Only the points later than 1999 June are connected since they better sample the lightcurve of IRAS $18576+0341$.

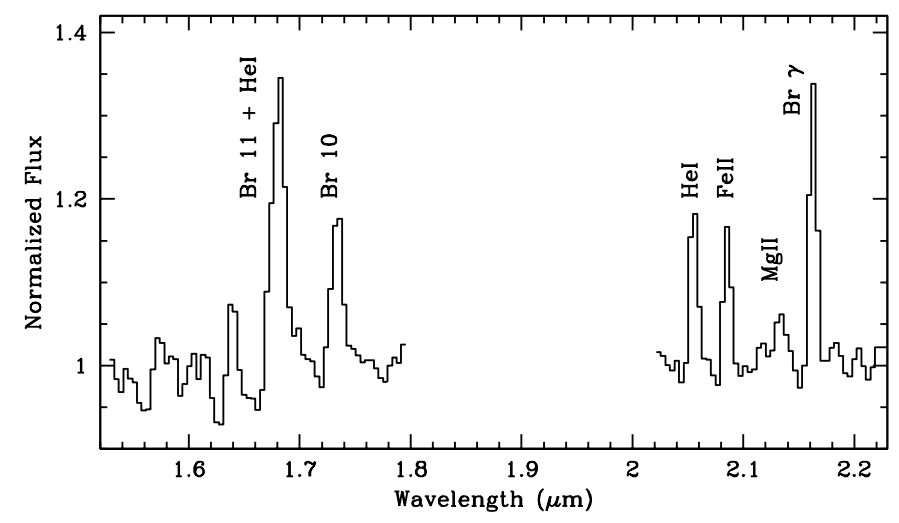

Fig. 4. The observed $J$-to- $K$ spectrum of IRAS $18576+0341$. The spectral resolution is $R \simeq 240$.

increased its $H$ and $K$ magnitudes by $\simeq 0.5$ mag with respect to the 2000 August minimum so that it is brightening again. With the exception of the highly uncertain 2000 August point, the $J$ lightcurve mimics those determined in $H$ and $K$. This variability is quite common among well known LBVs. For example, AG Carinae and HR Carinae vary by $\sim 2$ and 1 mag in $V$ respectively, over a period of $\sim 10$ years (Spoon et al. 1994).

\subsection{IR spectroscopy}

The infrared spectrum of IRAS $18576+0341$ is plotted in Fig. 4 where the continuum is normalized to unity and the 
spectral resolution is $R \simeq 240$. The most prominent emissions are due in turn to $\operatorname{Br} 11+\mathrm{HeI}, \operatorname{Br} \gamma, \mathrm{HeI} 2.06 \mu \mathrm{m}$, Br 10 and FeII $2.09 \mu \mathrm{m}$. The MgII doublet at $2.14 \mu \mathrm{m}$ can also be identified although it is much fainter.

It is interesting to compare the IRAS $18576+0341$ spectrum with the Morris et al. (1996) compilation of infrared spectra of evolved, massive stars. The FeII and MgII lines appear to be common among LBVs such as AG Carinae, WRAY 751 and He 3-519, so that their presence in the spectrum of IRAS $18576+0341$ strengthens the LBV classification of this star. The strength of the FeII $2.09 \mu \mathrm{m}$ line relative to HeI $2.06 \mu \mathrm{m}$ is particularly interesting: their ratio is as high as 1 . The largest FeII/HeI line ratio observed to date in a confirmed LBV is $\simeq 0.5$ in WRAY 751 (Morris et al. 1996), while a line ratio of $\simeq 1$ has been only observed in the spectrum of SGR 1806-20, a candidate LBV whose physical association with the soft-gamma repeater source is under debate (van Kerkwijk et al. 1995; Fuchs et al. 1999). On the other hand, the MgII lines in the spectrum of SGR 1806-20 appear to be stronger than the FeII $2.09 \mu \mathrm{m}$ feature, which is certainly not the case for IRAS $18576+0341$.

The $(\mathrm{Br} 11+\mathrm{HeI}) / \mathrm{Br} 10$ line ratio in the spectrum of IRAS $18576+0341$ is very similar to that observed in He 3-519, while the HeI 2.06/Br $\gamma$ ratio quite resembles the one observed in HDE 269582, an Ofpe/WN9 star also confirmed as an LBV by Humphreys \& Davidson (1994).

Ofpe/WN9 stars (Bohannan \& Walborn 1989, recently re-classified as WN9-11 by Crowther \& Smith 1997) are generally believed to be quiescent LBVs (Pasquali et al. 1997): AG Carinae is the typical example of an LBV whose spectrum shows the Ofpe/WN9 morphology when the star is at its minimum, hence at its maximum $T_{\text {eff }}$ (cf. Stahl 1987). He 3-519 is also characterised by an Ofpe/WN9like spectrum at its lightcurve minimum (Smith et al. 1994). Figure 3 shows that IRAS $18576+0341$ has just gone through its minimum and at this same epoch the star displays the spectral signatures of both LBV and Ofpe/WN9 stars in close similarity with He 3-519.

According to the stellar parameters derived by Crowther \& Smith (1997) and Pasquali et al. (1997), Ofpe/WN9 and WN9-11 stars have effective temperatures in the range between $21000 \mathrm{~K}$ and $35000 \mathrm{~K}$. Therefore, IRAS $18576+0341$ may be hotter than $15000 \pm 9000 \mathrm{~K}$ as estimated by Ueta et al. (2001).

\section{Conclusions}

We have observed IRAS $18576+0341$, a galactic LBV candidate, by means of infrared imaging and low-resolution spectroscopy. When compared with previously published photometry, our JHK magnitudes suggest a lightcurve with an amplitude of approximately 1.4 mag between 1999 June and 2001 August, suggesting a period of $2-3$ years. More continous monitoring is required to derive the true period of IRAS $18576+0341$. Established LBVs such as AG Carinae and HR Carinae are indeed characterised by an amplitude of 1-2 mag over somewhat larger period, $\simeq 10$ years. The first infrared spectrum ever taken of the star is characterised by prominent emissions due to $\mathrm{Br} 11+\mathrm{HeI}, \mathrm{Br} \gamma$ and HeI $2.06 \mu \mathrm{m}$. Also present are the Br 10, FeII $2.09 \mu \mathrm{m}$ and MgII $2.14 \mu \mathrm{m}$ lines. This spectral morphology is very similar to that observed for He 3-519, a galactic LBV also classified WN11 during its visual minimum. Therefore, our data place IRAS 18576+0341 among the Luminous Blue Variables and suggest that the star belongs to the WN11 class at its lightcurve minimum. Hence it may be hotter than $T_{\text {eff }}=15000 \pm 9000 \mathrm{~K}$ as computed by Ueta et al. (2001).

Further photometry and high-resolution spectroscopy of the star at infrared wavelengths are required to accurately derive the lightcurve and the stellar parameters (i.e. mass loss rate) of IRAS $18576+0341$.

Acknowledgements. We would like to thank the referee for his/her valuable comments and suggestions.

\section{References}

Bessell, M. S., \& Brett, J. M. 1988, PASP, 100, 1134

Bohannan, B., \& Walborn, N. R. 1989, PASP, 101, 520

Crowther, P. A., \& Smith, L. J. 1997, A\&A, 320, 500

Figer, D. F., McLean, I. S., \& Morris, M. 1999, ApJ, 514, 202

Fuchs, Y., Mirabel, F., Chaty, S., et al. 1999, A\&A, 350, 891

Garcia-Lario, P., Manchado, A., Pych, W., \& Pottasch, S. R. 1997, A\&AS, 126, 479

Geballe, T. R., Najarro, F., \& Figer, D. F. 2000, ApJ, 530, L97 Humphreys, R. M., \& Davidson, K. 1994, PASP, 106, 1025

Langer, N., \& Heger, A. 1998, in B[e] Stars, ed. A. M. Hubert, \& C. Jaschek, ASSL, 233, 235

Langer, N., Hamann, W.-R., Lennon, M., et al. 1994, A\&A, 290, 819

Morris, P. W., Eenens, P. R. J., Hanson, M. M., Conti, P. S., \& Blum, R. D. 1996, ApJ, 470, 597

Oliva, E., \& Origlia, L. 1992, A\&A, 254, 466

Pasquali, A., Langer, N., Schmutz, W., et al. 1997, ApJ, 478, 340

Persson, S. E., Murphy, D. C., Krzeminski, W., Roth, M., \& Rieke, M. J. 1998, AJ, 116, 247

Rieke, G. H., \& Lebofsky, M. J. 1985, ApJ, 288, 618

Smith, L. J., Crowther, P. A., \& Prinja, R. K. 1994, A\&A, 281, 833

Smith, L. J., Nota, A., Pasquali, A., et al. 1998, ApJ, 503, 278 Spoon, H. W. W., de Koter, A., Sterken, C., Lamers, H. J. G. L. M., \& Stahl, O. 1994, A\&AS, 106, 141

Stahl, O. 1987, A\&A, 182, 229

Stetson, P. B. 1987, PASP, 99, 191

Ueta, T., Meixner, M., Dayal, A., et al. 2001, ApJ, 548, 1020 van Kerkwijk, M. H., Kulkarni, S. R., Matthews, K., \& Neugebauer, G. 1995, ApJ, 444, L33

Voors, R. H. M., Geballe, T. R., Waters, L. B. F. M., Najarro, F., \& Lamers, H. J. G. L. M. 2000, A\&A, 362, 236 